\title{
A REMARK ON THE GENERALIZED NUMERICAL RANGE OF A NORMAL MATRIX
}

\author{
by YIK-HOI AU-YEUNG and FUK-YUM SING
}

(Received 1 April, 1976)

1. Introduction. Let $A$ be an $n \times n$ complex normal matrix and let $\mathscr{W}(A)=\left\{\operatorname{diag} U A U^{*}\right.$ : $U$ is unitary) where $U^{*}$ is the conjugate transpose of $U$. It is known that $\mathscr{W}(A)$ may not be convex $[1,3]$ and it is convex when $A$ is Hermitian $[1,2]$. In this note we show that $\mathscr{W}(A)$ is convex if and only if the eigenvalues of $A$ are collinear (i.e. there exist complex numbers $\alpha(\neq 0)$ and $\beta$ such that $\alpha A+\beta I$ is Hermitian).

Hence for most normal matrices $A, \mathscr{W}(A)$ is not convex.

\section{Generalized numerical range.}

LEMMA 1. Let $Q=\left(\begin{array}{ll}U & a \\ b^{*} & \mu\end{array}\right)$ be an $(n+1) \times(n+1)$ unitary matrix, where $U$ is an $n \times n$ matrix. Then there exists a real number $\gamma$ such that $U+\gamma a b^{*}$ is unitary.

Proof. Using the property that $U b+\bar{\mu} a=0$ and $b^{*} b+\mu \bar{\mu}=1$, we can show that $\left(U+\gamma a b^{*}\right)$ $\left(U+\gamma a b^{*}\right)^{*}=U U^{*}+\left[\gamma^{2}(1-\mu \bar{\mu})-\gamma(\mu+\bar{\mu})\right] a a^{*}$. Since $1-\mu \bar{\mu} \geqq 0$, it is possible to find $\gamma$ so that $\left[\gamma^{2}(1-\mu \bar{\mu})-\gamma(\mu+\bar{\mu})\right] a a^{*}=a a^{*}$ (when $\mu \bar{\mu}=1, a=0$ ). Since $U U^{*}+a a^{*}=I_{n}$, where $I_{n}$ is the $n \times n$ identity matrix, the lemma is proved.

Proposition 2.

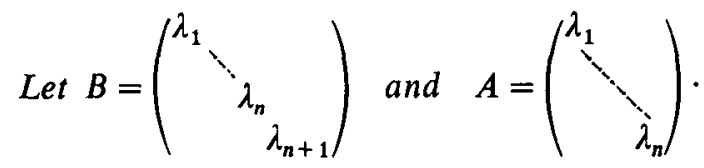

If $\mathscr{W}(B)$ is convex and $\lambda_{n+1}$ is a vertex of the convex hull of the points $\lambda_{1}, \ldots, \lambda_{n+1}$, then $\mathscr{W}(A)$ is convex.

Proof. For any $n \times n$ unitary matrices $U_{1}$ and $U_{2}$ and $0 \leqq \alpha \leqq 1$ we can find unitary matrix $Q=\left(\begin{array}{cc}U & a \\ b^{*} & \mu\end{array}\right)$ such that

$\operatorname{diag}\left\{\alpha\left(\begin{array}{ll}U_{1} & 0 \\ 0 & 1\end{array}\right)\left(\begin{array}{ll}A & 0 \\ 0 & \lambda_{n+1}\end{array}\right)\left(\begin{array}{cc}U_{1}^{*} & 0 \\ 0 & 1\end{array}\right)+(1-\alpha)\left(\begin{array}{ll}U_{2} & 0 \\ 0 & 1\end{array}\right)\left(\begin{array}{ll}A & 0 \\ 0 & \lambda_{n+1}\end{array}\right)\left(\begin{array}{ll}U_{2}^{*} & 0 \\ 0 & 1\end{array}\right)\right\}=\operatorname{diag} Q B Q^{*}$.

Hence we have

$$
\begin{gathered}
b^{*} A b+\lambda_{n+1} \mu \bar{\mu}=\lambda_{n+1}, \\
\operatorname{diag}\left(\alpha U_{1} A U_{1}^{*}+(1-\alpha) U_{2} A U_{2}^{*}\right)=\operatorname{diag}\left(U A U^{*}+\lambda_{n+1} a a^{*}\right) .
\end{gathered}
$$

From (1), since $\lambda_{n+1}$ is a vertex and $b^{*} b+\mu \bar{\mu}=1$, it follows that if $b^{*}=\left(b_{1}, \ldots, b_{n}\right)$, we have

Glasgow Math. J. 18 (1977) 179-180. 
$b_{i}=0$ for all $i$ such that $\lambda_{i} \neq \lambda_{n+1}$. This implies that $b^{*} A=\lambda_{n+1} b^{*}$ and $A b=\lambda_{n+1} b$. We can then obtain

$$
\begin{aligned}
& \left(U+\gamma a b^{*}\right) A\left(U+\gamma a b^{*}\right)^{*} \\
= & U A U^{*}+\lambda_{n+1}\left[\gamma^{2}(1-\mu \bar{\mu})-\gamma(\mu+\bar{\mu})\right] a a^{*} \\
= & U A U^{*}+\lambda_{n+1} a a^{*},
\end{aligned}
$$

where $\gamma$ is chosen as in Lemma 1. From (2) we see that $\mathscr{W}(A)$ is convex.

THEOREM 3.

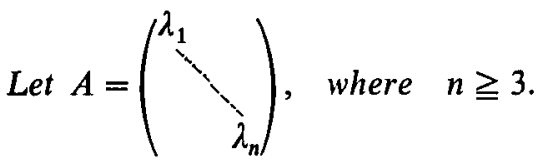

Then $\mathscr{W}(A)$ is convex if and only if $\lambda_{1}, \ldots, \lambda_{n}$ are collinear.

Proof. First we prove sufficiency. When the eigenvalues of $A$ are collinear, $\alpha A+\beta I$ is Hermitian for some complex numbers $\alpha(\neq 0)$ and $\beta$. Since $\mathscr{W}(\alpha A+\beta I)$ is convex, it follows that $\mathscr{W}(A)$ is also convex. To prove necessity we use induction on $n$. The case $n=3$ is proved in [1]. Suppose $\mathscr{W}(A)$ is convex and the eigenvalues of $A$ are $\lambda_{1}, \ldots, \lambda_{n}$ where $n>3$. If the eigenvalues are not collinear, we take a vertex, say $\lambda_{1}$. By Proposition 2 and the induction assumption, $\lambda_{2}, \lambda_{3}, \ldots, \lambda_{n}$ are collinear. Consider a vertex on this line segment, say $\lambda_{2}$. By the same argument, $\lambda_{1}, \lambda_{3}, \ldots, \lambda_{n}$ are collinear. We must have then $\lambda_{3}=\lambda_{4}=\ldots=\lambda_{n}$. (For if $\lambda_{i} \neq \lambda_{j}$ for some $i, j \geqq 3$, then $\lambda_{1}, \lambda_{i}, \lambda_{j}$ are not collinear.) This implies $\lambda_{3}$ is a vertex and hence $\lambda_{1}, \lambda_{2}, \lambda_{4}$ are collinear, which gives a contradiction. Therefore the eigenvalues must be collinear.

From Theorem 3 we have immediately the following result.

COROLlaRY 4. If $A=\left(\begin{array}{c}A_{1} \\ A_{2}\end{array}\right)$ is a normal matrix and $\mathscr{W}(A)$ is convex, then $\mathscr{W}\left(A_{1}\right)$ and $\mathscr{W}\left(A_{2}\right)$ are convex.

\section{REFERENCES}

1. P. A. Fillmore and J. P. Williams, Some convexity theorems for matrices, Glasgow Math. J. 12 (1971), 110-116.

2. A Horn, Doubly stochastic matrices and the diagonal of a rotation matrix, Amer. J. Math. 76 (1954), 620-630.

3. L. E. Lerer, On the diagonal elements of normal matrices (Russian), Mat. Issled. 2 (1967), $156-163$.

DePartment of Mathematics

UNIVERSITY OF HONG KONG

PRINTED IN GREAT BRITAIN BY ROBERT MACLEHOSE AND CO. LTD

PRINTERS TO THE UNIVERSITY OF GLASGOW 